\title{
Reactive oxygen species modulator 1 regulates oxidative stress and induces renal and pulmonary fibrosis in a unilateral ureteral obstruction rat model and in HK-2 cells
}

\author{
DONGHAI LIU, YING LIU, ZHENKUN XIA, HAIYUN DONG and ZHUWEN YI
}

Department of Pediatrics, The Second Xiangya Hospital of Central South University, Changsha, Hunan 410011, P.R. China

Received September 2, 2016; Accepted May 19, 2017

DOI: $10.3892 / \mathrm{mmr} .2017 .7161$

\begin{abstract}
Renal interstitial fibrosis (RIF) is the main process that leads to renal failure. It is necessary to investigate the mechanism of RIF and identify appropriate methods of regulating it. Furthermore, unilateral ureteral obstruction is a frequently used model for the study of RIF. The morphological damage associated with kidney and lung dysfunction was detected using histopathological experiments. Subsequently, high expression of reactive oxygen species (ROS) modulator 1 (ROMO1) and ROS was measured in blood serum. In addition, epithelial-mesenchymal transition marker, transforming growth factor $\beta$ (TGF- $\beta$ ) and mothers against decapentaplegic homolog $2 / 3$ expression was evaluated using the reverse transcription-quantitative polymerase chain reaction and western blotting. All serious symptoms were relieved to a certain extent following oxidation inhibitor intervention using three common antioxidants. HK-2 cells were treated with $\mathrm{H}_{2} \mathrm{O}_{2}$ to cause oxidative stress, and ROMO1 and fibrosis marker expression increased; however, activation was suppressed byROMO1 knockout. The present study provides evidence that the expression of ROMO1 induces ROS production and activates the TGF- $\beta$ signaling pathway. It may be concluded that ROMO1 helps to provide a molecular basis for improved clinical intervention and prognosis of patients.
\end{abstract}

\section{Introduction}

Renal interstitial fibrosis (RIF) is the primary pathological mechanism of a variety of kidney diseases and leads to the end-stage renal diseases. Epithelial-mesenchymal transition

Correspondence to: Professor Zhuwen Yi, Department of Pediatrics, The Second Xiangya Hospital of Central South University, 139 Renmin Road, Changsha, Hunan 410011, P.R. China E-mail: zhuwenyi2016@163.com

Key words: reactive oxygen species modulator 1, unilateral ureteral obstruction, HK-2, reactive oxygen species, transforming growth factor $\beta$, oxidative stress
(EMT) is the major process of cell fibrosis $(1,2)$. A previous study indicated that EMT-associated markers were highly expressed in kidney disease (3). Transforming growth factor $\beta$ (TGF- $\beta$ ), an essential factor which induces EMT through mothers against decapentaplegic homolog (Smad) pathway activation, serves an important role in RIF (4).

Reactive oxygen species (ROS) are essential for cell homeostasis, cell survival and gene expression. Recent research demonstrated that the production of intracellular ROS was regulated by ROS modulator 1 (ROMO1) (5). Upregulation of ROS and ROMO1 is typically observed in cancer cells. For instance, the level of ROS in Fanconi anemia cells was modulated by ROMO1 through an EMT-associated signaling pathways (6). Furthermore, the expression of ROS in rat brain astrocyte cells also influences the level of TGF- $\beta 1$ through an EMT-associated signaling pathway (7). In addition, the level of ROS in lung epithelial cell mitochondria was persistently induced by TGF- $\beta 1$. Therefore, it is hypothesized that ROMO1 may effectively induce the expression of endogenous ROS and increase oxidative stress via activation of the TGF- $\beta$ signaling pathway, which leads to EMT activity and exacerbates the RIF process.

Unilateral ureteral obstruction (UUO) is a well-established animal model which is utilized in the study of renal tubulointerstitial fibrosis. The model, leads to an obstruction in the renal drainage system, and causes acute renal function damage and chronic structural damage to the kidney (8). Furthermore, a reduced renal blood flow, interstitial macrophage infiltration $(9,10)$ and fibroblast proliferation result in RIF-associated renal function failure (11). The model is simple to build and repeat, which allows the convenient study of renal cell transdifferentiation. Chronic kidney disease affects the water balance in the lung and kidney disease associated with oxidative stress typically causes damage to the lung (12). Therefore, an improved understanding of the lung damage in kidney disease may help to improve clinical intervention and prognosis inpatients.

In the present study, a UUO rat model was established. The tissue damage at the obstruction side of the kidney was observed, and the expression of ROS and ROMO1 were investigated. Furthermore, to identify the regulatory mechanism of ROMO1 the associated pathway factors, including TGF- $\beta 1$, fibronectin (FN) and EMT markers, were examined in the present study. 


\section{Materials and methods}

Drugs and reagents. Oxidation inhibitor N-acetyl-L-cysteine (NAC), tempol and apocynin were purchased from Sigma-Aldrich (Merck KGaA, Darmstadt, Germany), Santa Cruz Biotechnology, Inc. (Dallas, TX, USA) and Selleck Chemicals (Houston, TX, USA), respectively. Antibodies used in the western blotting were purchased from Aura Biosciences, Inc. (Cambridge, MA, USA), as well as the radioimmunoprecipitation assay (RIPA) buffer, phenylmethylsulfonyl fluoride (PMSF), SDS-PAGE kit, protease inhibitors and phosphatase inhibitors.

Animal materials. A total of 100 male Sprague-Dawley rats aged 7 weeks weighing 180-200 g were obtained from the Second Xiangya Hospital of Central South University (Changsha, China). Where relevant, the experiment conforms to the national guidelines for animal usage in research $(13,14)$. All the experimental animals were housed with free access to food and water, and maintained with a 12-h light/dark cycle (lights on at 7:00 a.m.), at $22^{\circ} \mathrm{C}$ and low humidity.

Rat modeling. A UUO rat model was established in the present study. The left ureter was ligated in 808 -week-old male rats prior to being divided into four groups, consisting of the model, model+tempol, model+apocynin and model+NAC groups. The three treatment groups received an intraperitoneal injection of $20 \mathrm{mg} / \mathrm{kg} / \mathrm{day}$ tempol, $10 \mathrm{mg} / \mathrm{kg} / \mathrm{day}$ apocynin and $300 \mathrm{mg} / \mathrm{kg} /$ day NAC, respectively. The unoperated 20 rats were treated as the control group, and the control and model groups received water in place of the treatment. The experiment lasted for 21 days. The data and tissues were collected on the 1st, 7th, 14th and 21st days. All animal treatments and experiments were approved by the Institutional Animal Care and Use Committee of the Second Xiangya Hospital of Central South University.

Surgery. Rats were anesthetized with $4 \mathrm{ml} / \mathrm{kg} 10 \%$ chloralhydrate. The blood samples were collected by the abdominal aortic method and kept in tubes containing EDTA and centrifuged at $2,500 \mathrm{~g}$ for $15 \mathrm{~min}$ at $4^{\circ} \mathrm{C}$. Serum was subsequently collected and stored at $-80^{\circ} \mathrm{C}$ until analysis. Kidneys and lungs were removed surgically and cut into two parts; one part was stored at $-80^{\circ} \mathrm{Cuntil}$ RNA or protein extraction, and the other part was kept in $4 \%$ formalin and stored at $4^{\circ} \mathrm{C}$ until tissue dehydration embedding. The urine in the bladder was collected for the urinary creatinine test.

Biochemical test and analysis. Serum was stored at $-80^{\circ} \mathrm{C}$ following surgery. The creatinine content in the serum and urine, as well as total protein, blood urea nitrogen (BUN) and uric acid (UA) content, were measured using respective test kits (Changchun Huili Biotechnology Co., Ltd., Changchun, China) and a semiautomatic biochemistry analyzer. The ROS assay kit obtained from Beyotime Institute of Biotechnology (Haimen, China) was used to detect ROS. Creatinine clearance was calculated as follows: Creatinine clearance rate $(\mathrm{ml} / \mathrm{min} / \mathrm{kg})=$ [urinary creatinine $(\mu \mathrm{mol} / \mathrm{l}) \times 24 \mathrm{~h}$ urine volume $(\mathrm{ml})] /[$ serum creatinine $(\mu \mathrm{mol} / \mathrm{l}) \times(1,000 /$ body weight (g) $x(1 / 1440)]$.
Cell culture. The HK-2 renal epithelial cell line was purchased from the American Type Culture Collection (ATCC; Manassas, VA, USA) and characterized according to ATCC instructions. HK-2 cells were cultured in RPMI 1640 medium (Hyclone, GE Healthcare Life Sciences, Logan, UT, USA) supplemented with $10 \%$ fetal bovine serum at $37^{\circ} \mathrm{C}$ in an atmosphere containing $5 \% \mathrm{CO}_{2}$. Cells were grown to $80 \%$ confluence and transfected with the ROMO1 clustered regularly interspaced short palindromic repeats (CRISPR)/CRISPR-associated endonuclease Cas9 (Cas9) knockout (KO) plasmid or control CRISPR/Cas9 plasmid (Santa Cruz Biotechnology, Inc.) using Lipofectamine 2000 (Invitrogen; Thermo Fisher Scientific, Inc., Waltham, MA, USA), according to the manufacturer's protocol. The HK-2 original strain was treated with $\mathrm{H}_{2} \mathrm{O}_{2}$ $(50 \mu \mathrm{M})$ for a time gradient $(0.5,1$ and $2 \mathrm{~h})$. Then, transfected cells were treated with $\mathrm{H}_{2} \mathrm{O}_{2}$ for $2 \mathrm{~h}$ prior to being treated with NAC $(10 \mathrm{mM})$. The whole-cell proteins were extracted for western blotting.

Reverse transcription-quantitative polymerase chain reaction $(R T-q P C R)$. The expression level of mRNA was measured by RT-qPCR. The samples were kept at $-80^{\circ} \mathrm{C}$ prior to RNA extraction using TRIzol (Invitrogen; Thermo Fisher Scientific, Inc.). The Revert Aid ${ }^{\mathrm{TM}}$ First Stand cDNA Synthesis kit (Thermo Fisher Scientific, Inc.) was utilized for reverse transcription into cDNA. RT-qPCR reactions were performed using a Real-Time PCR Quantification system (ABI 7300; Applied Biosystems; Thermo Fisher Scientific, Inc.). The following thermocycling conditions were used: Initial denaturation at $95^{\circ} \mathrm{C}$ for $3 \mathrm{~min}$; 40 cycles of denaturation at $95^{\circ} \mathrm{C}$ for $10 \mathrm{sec}$ and annealing at $60^{\circ} \mathrm{C}$ for $30 \mathrm{sec}$. Each sample reaction was performed in triplicate. Relative expression levels were calculated using the $2^{-\Delta \Delta C q}$ method (15). The primers for ROMO1, FN, TGF- $\beta 1$ and GAPDH were as follows: ROMO1 sense, TGT CTC AGG ATC GGA ATG CG and antisense, GGC CAT GAA AGT GCC AAA GG; FN sense, CGT CCC ACG ATC CGA TGA and antisense, CTT GCT TTC CCT GCC CTG A; TGF- $\beta 1$ sense, TGA ACC AAG GAG ACG GAA TAC AGG G and antisense, GCC ATG AGG AGC AGG AAG GGT C and GAPDH sense, TAC CAG GGC TGC CTT CTC TTG and antisense, CGG GAT CTC GCT CCT GGA AG.

Western blotting analysis. The kidney and lung tissues were removed surgically. The whole-cell extract proteins were prepared for western blotting and western blotting was performed as previously described (16). The kidneys were homogenized in 1:9 RIPA lysis buffer (Auragene, Changsha, Hunan, China). The proteins were subsequently electrophoretically resolved on 15 and 6\% SDS-PAGE gels, and transferred to nitrocellulose membranes. The membranes were blocked with skimmed milk at room temperature for $1 \mathrm{~h}$, and incubated withanti-ROMO1 (cat. no. SAB2107329; Sigma-Aldrich; Merck KGaA; 1:500), anti-FN (cat. no. AM2784; Abzoom Biolabs, Inc., Dallas, TX, USA; 1:1,000), anti-epithelial (E)-cadherin (cat. no. ab76055; Abcam, Cambridge, MA, USA; 1:500), anti-vimentin (cat. no. ab8978; Abcam; 1:500), anti-TGF- $\beta 1$ (cat. no. ab66043; Abcam, 1:800), anti-Smad2/3 (cat. no. AM4060; Abzoom Biolabs, Inc.; 1:800), anti-phosphorylated (p)-Smad2/3 (cat. no. 8828S; Cell Signaling Technology, Inc., Danvers, 


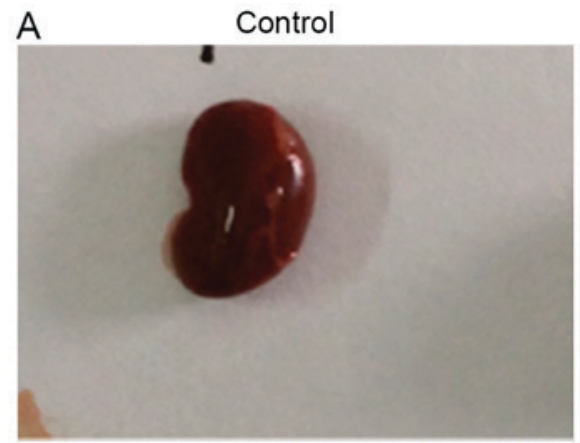

B



C

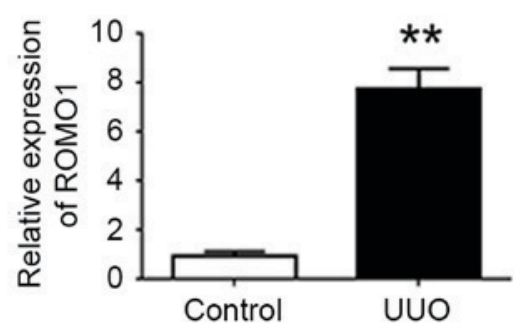

D


UUO

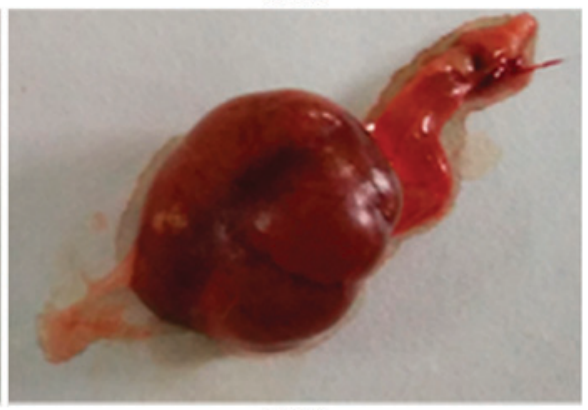

UUO

Figure 1. UUO model leads to hydronephrosis and an increased expression of ROMO1. (A) Ligation in one side of kidney caused a serious lesion when compared with control. (B) Hematoxylin and eosin staining indicated that there were serious pathological alterations in ligated side of kidney. The (C) mRNA and (D) protein expression levels of ROMO1 were increased in the damaged kidney. $\mathrm{n}=5$. ${ }^{* *} \mathrm{P}<0.01$ vs. the control. ROMO1, reactive oxygen species modulator 1; UUO, unilateral ureteral obstruction.

MA, USA; 1:800) and anti-GAPDH (cat. no. LCA03; Aura Biosciences, Inc., Cambridge, MA, USA; 1:1,500) antibodies at $4^{\circ} \mathrm{C}$ overnight. Membranes were subsequently incubated with thegoat anti-mouse immunoglobulin (Ig)G [heavy chain and light chain $(\mathrm{H}+\mathrm{L})]$ horseradish peroxidase (HRP)-conjugated (cat. no. SA001; Aura Biosciences, Inc.; 1:15,000) and goat anti-rabbit IgG $(\mathrm{H}+\mathrm{L}) \mathrm{HRP}$-conjugated (cat. no. SA009; Aura Biosciences, Inc.; 1:15,000) secondary antibodies at room temperature $40 \mathrm{~min}$. The signals were visualized using an Enhanced Chemiluminescence kit (Aura Biosciences, Inc.). Image Pro-Plus version 6.0 software (Media Cybernetics, Inc., Rockville, MD, USA) was used for densitometric analysis.

Histopathology. The kidney and lung tissues were removed surgically. Tissues were fixed in $4 \%$ formalin at $4^{\circ} \mathrm{C}$ for $12-24 \mathrm{~h}$ prior to being dehydrated, paraffin-embedded and sliced into $3 \mu \mathrm{m}$ sections. Sections were deparrafinized in xylene at room temperature for $10 \mathrm{~min}$ twice. The method for hematoxylin and eosin (HE) staining of the aortic tissues was conducted as previously described (17). The staining results were observedundera AE31light microscope (Motic Deutschland $\mathrm{GmbH}$, Wetzlar, Germany) at a magnification of x100.

Statistical analysis. The data are presented as the mean \pm standard error of the mean. The data were analyzed using the SPSS software (version 11.0; SPSS Inc., Chicago, IL, USA). The Student's $t$ test was used to analyze the differences between two groups and one-way analysis of variance followed by Dunnett's post hoc test was used to analyze the differences among three or more groups. $\mathrm{P}<0.05$ was considered to indicate a statistically significant difference.

\section{Results}

UUO model leads to pathological alterations and an increase in ROMO1 expression. In the UUO model, the left ureter was ligated. In order to detect the influence of the ligation in the kidney, the ligated renal tissues were collected following surgery. Fig. 1A and B demonstrates that there was pyelectasis in the ligated side, while the kidneys in the control group were healthy without any apparent damage. Fig. 1C and D 
A

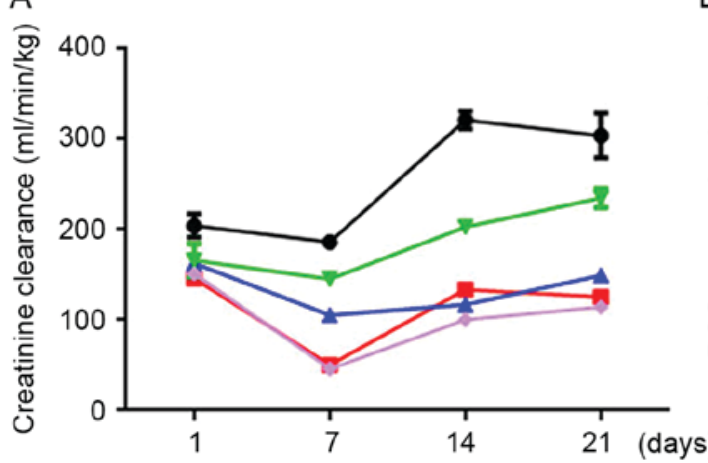

C

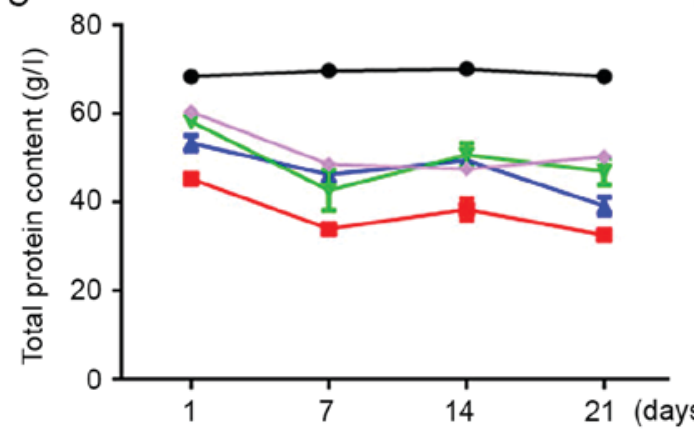

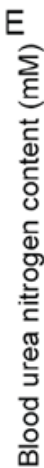

B

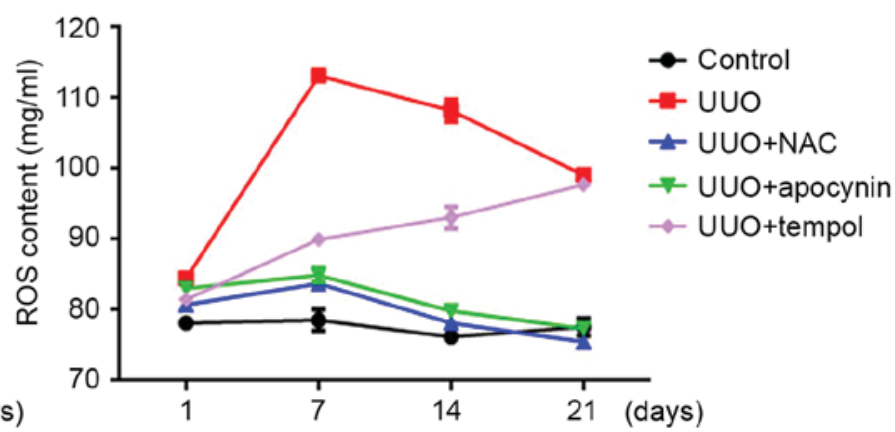

D

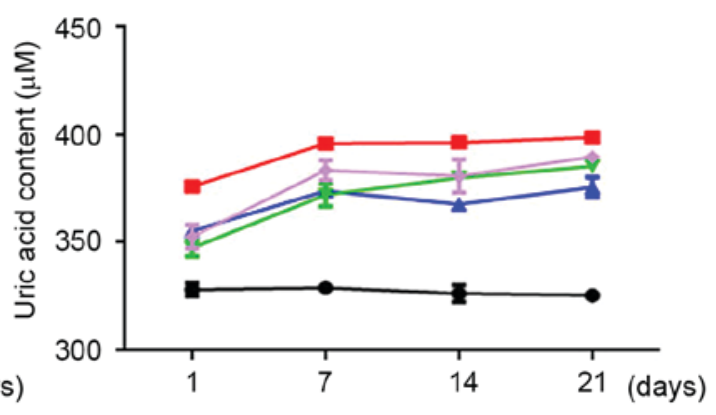

$\mathrm{F}$

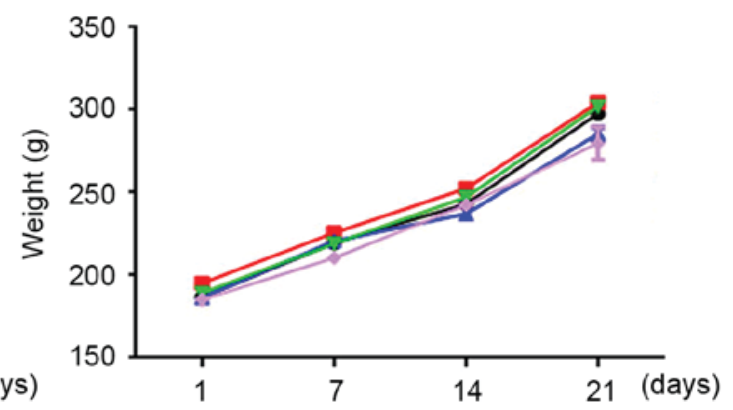

Figure 2. Detection of biochemical indexes in the UUO rat model. The UUO model exhibited decreased (A) creatinine clearance, (B) increased ROS, (C) decreased total protein, (D) increased uric acid, (E) increased blood urea nitrogen and (F) no difference in body weight. UUO, unilateral ureteral obstruction; ROS, reactive oxygen species; NAC, N-acetyl-L-cysteine.

demonstrate that ROMO1 mRNA and protein expression was significantly increased in the UUO model.

Creatinine clearance rate, ROS level and kidney function was disordered under oxidative stress. The level of creatinine clearance and ROS are presented in Fig. 2. The clearance rate of creatinine was decreased in the UUO model compared with the control, and treatment with tempol and NAC exhibited no effect on this decrease. By contrast, treatment with Apo decreased the effect of UUO on creatinine clearance (Fig. 2A). ROS was increased in the UUO group compared with the control; however, this increase was reduced following treatment with NAC and Apo (Fig. 2B).

In order to measure renal function, the content of total protein, BUN and UA were detected. As demonstrated in Fig. 2C-E, there were slight positive therapeutic effects following treatment with tempol, Apo and NAC in the short term, when compared with model group. Furthermore, body weight increased linearly throughout the 21 days of the experiment in all of the groups (Fig. 2F). the results infer that there is no influence on body weight following ureteral ligation or oxidation inhibitor intervention.

ROMO1 stimulates the TGF- $\beta 1$ signaling pathway, causes EMT and extracellular matrix (ECM) generation. mRNA expression of ROMO1, FN and TGF- $\beta 1$ in the kidney and lung were detected by real time PCR, and the protein levels, including Smad2/3, E-cadherin and vimentin, were measured by western blotting (Fig. 3).

ROMO1 mRNA levels altered on the 1st day of treatment when compared with the model. The ROMO1 mRNA expression level in the UUO model was increased by $>12$-fold compared with the control and increased by $\sim 2$-fold compared with the treatment groups on the 14th day. FN mRNA was also increased in the UUO model when compared with the control group; however, this increase was reduced by the treatment interventions. On the 14th day the expression of FN in the model group was increased by $\sim 2$-fold when 

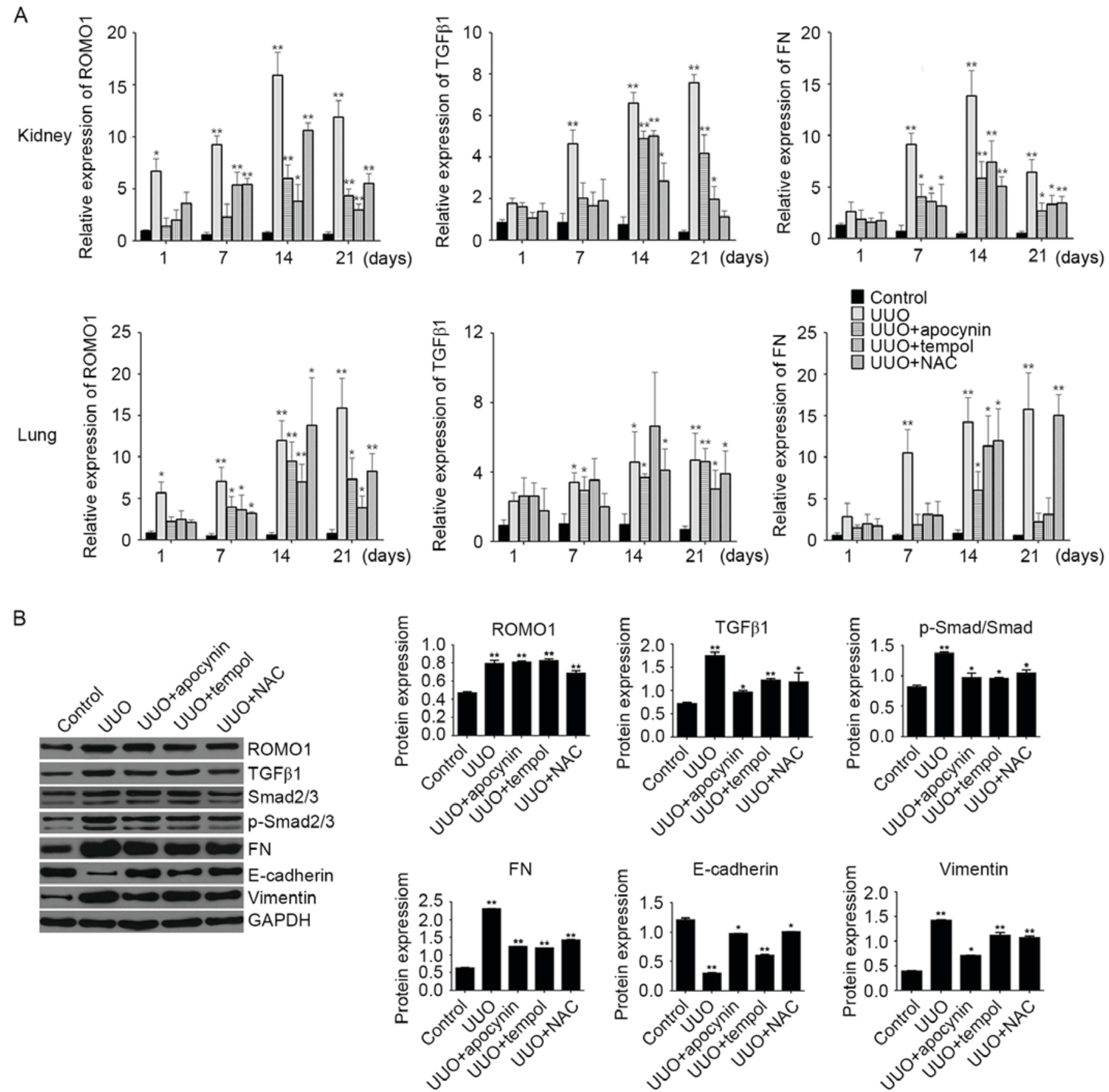

Figure 3. ROMO1 stimulates the TGF- $\beta$ signaling pathway and epithelial-mesenchymal transition. (A) mRNA expression of ROMO1, FN and TGF- $\beta 1$ following UUO. (B) Protein expression of ROMO1, FN, TGF- $\beta 1$, p-Smad/Smad, E-cadherin and vimentin following UUO. $n=5$. ${ }^{*} \mathrm{P}<0.05$, ${ }^{* *} \mathrm{P}<0.01$ vs. the control. ROMO1, reactive oxygen species modulator 1; TGF, transforming growth factor; FN, fibronectin; UUO, unilateral ureteral obstruction; p, phosphorylated; Smad, mothers against decapentaplegic homolog; E, epithelial; NAC, N-acetyl-L-cysteine.

compared with the treatment groups. Furthermore, TGF- $\beta 1$ mRNA expression was increased in the UUO model group by 7-foldcompared with the control group on day 14 .

In order to further investigate the TGF- $\beta 1$ signaling pathway and EMT in the UUO group. The protein expression of TGF $\beta 1, \mathrm{p}$-Smad2/3, $\operatorname{Smad} 2 / 3, \mathrm{E}$-cadherin and vimentin were detected by western blotting. The level of E-cadherin was downregulated in the UUO model, while this effect was partially reversed in the Apo, tempol and NAC groups. By contrast, other proteins were highly expressed in the UUO model, but reduced in the oxidation inhibitor groups (Fig. 3B).
Treatment with tempol, Apo and NAC alleviates kidney and lung damage. The pathological alterations in the kidney and lung were detected by HE staining. As presented in Fig. 4, renal interstitial hemorrhage occurred in the kidney following UUO. Vacuolar degeneration, inflammatory cell infiltrate and cell edema were observed in the model group, and it was diagnosed as chronic nephritis with cystic lesions. The damage was markedly improved following treatment with tempol, Apo and NAC on the 14th day. The treatment efficacy was as follows: NAC $<$ tempol $<\mathrm{APO}$. In the lungs, hyperplasia of fibrous tissues was observed in the model group, as well as inflammatory cell infiltrate and bronchiectasis 


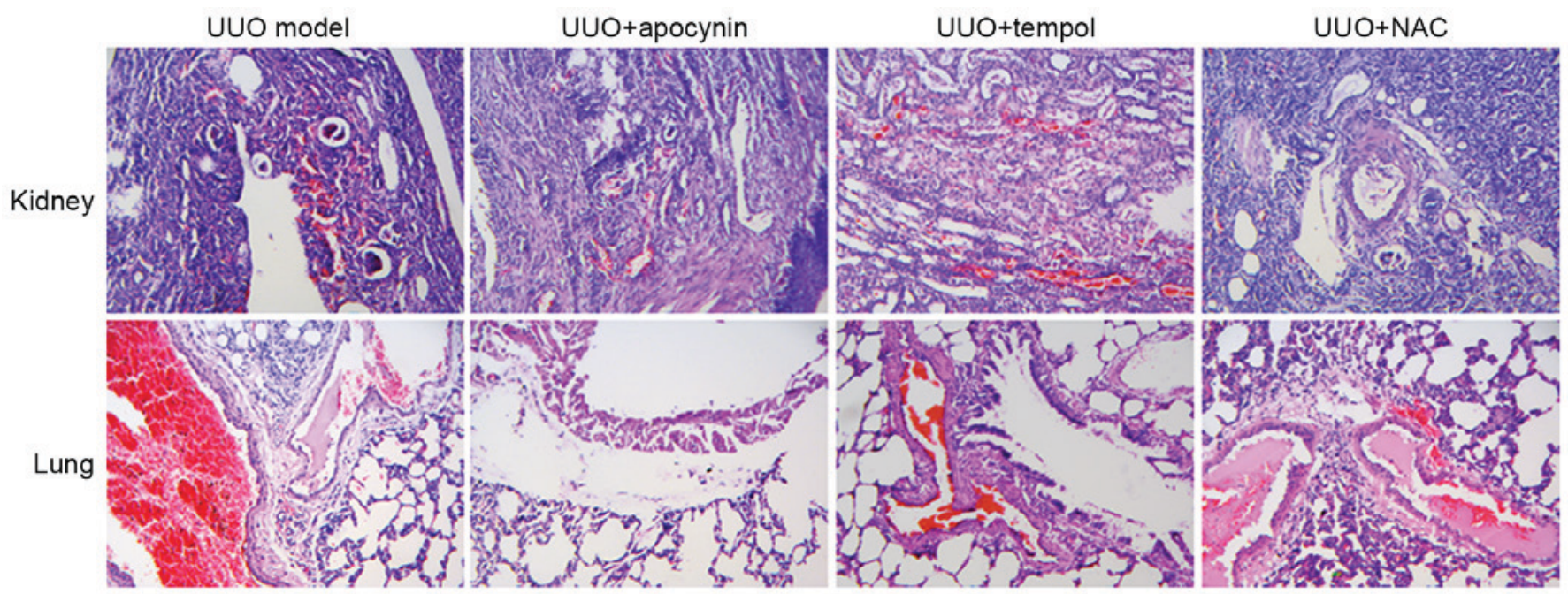

Figure 4. Morphological alterations in the kidney and lung were evaluated by histopathological staining. Clear lesions were observed in the UUO model in the kidney and lung; however, were improved by oxidation inhibitor intervention. UUO, unilateral ureteral obstruction; NAC, N-acetyl-L-cysteine.

A

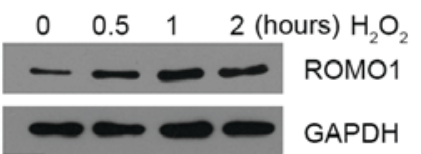

C
B

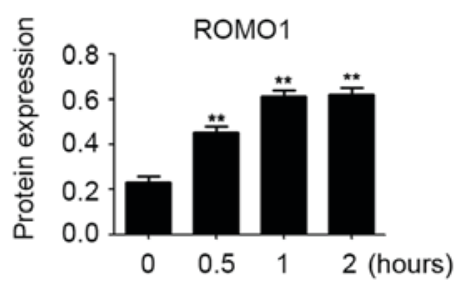

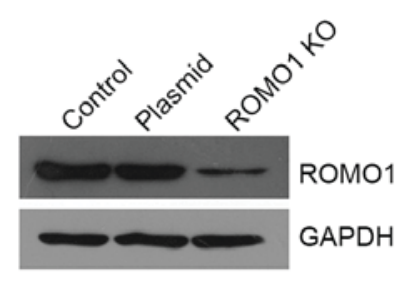
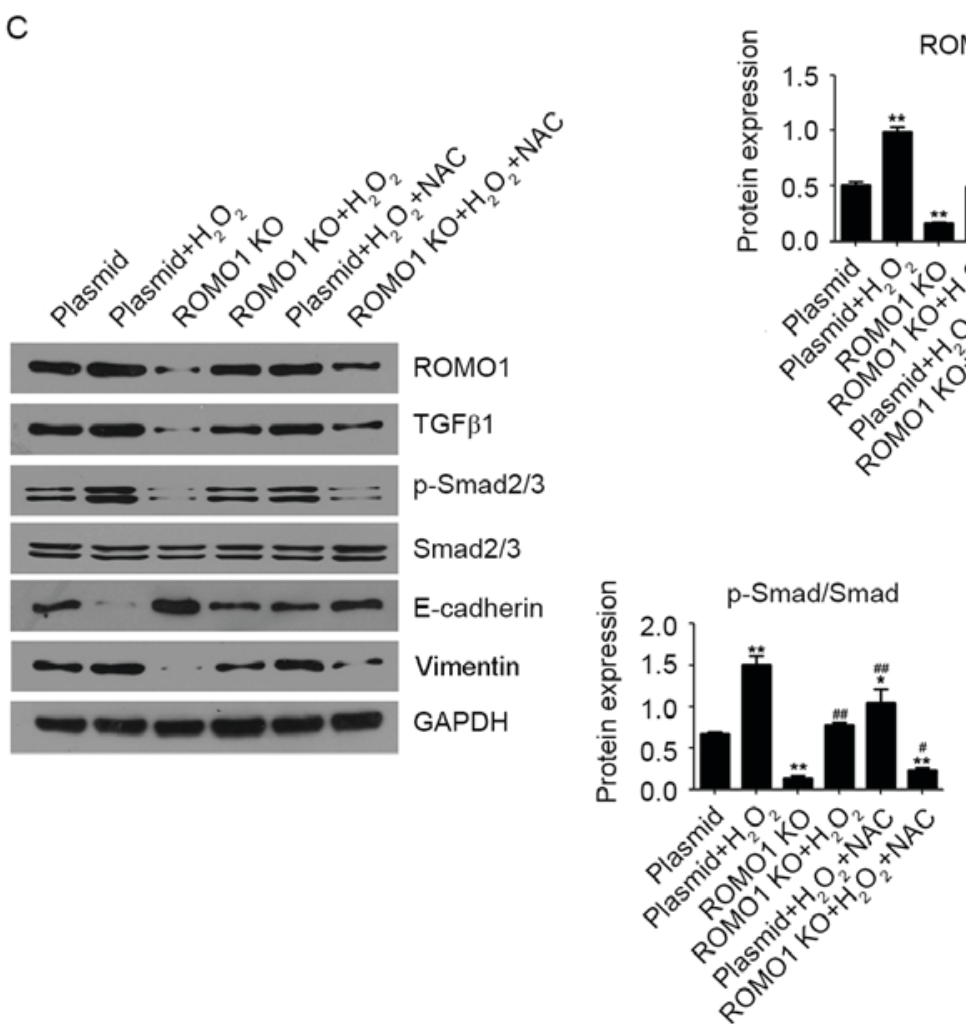
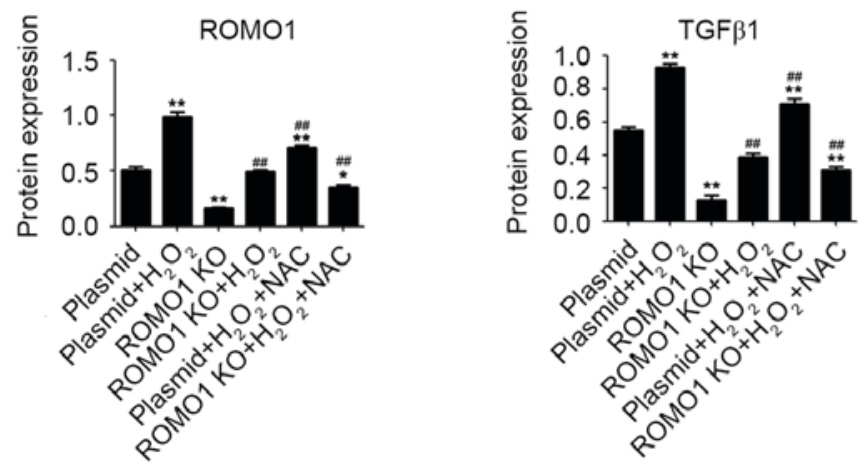

Figure 5. Cell fibrosis is suppressed during oxidative stress following ROMO1 KO. (A) The level of ROMO1 was analyzed by western blotting and was demonstrated to increase when cells were treated with $\mathrm{H}_{2} \mathrm{O}_{2}$. (B) The expression of ROMO1 was analyzed by western blotting and was demonstrated to decrease when cells were treated with the ROMO1-KO plasmid. (C) TGF- $\beta$ signaling pathway activation and epithelial-mesenchymal transition was reduced following ROMO1 KO, as analyzed by western blotting. $\mathrm{n}=3$. ${ }^{*} \mathrm{P}<0.05,{ }^{* *} \mathrm{P}<0.01$ vs. $0 \mathrm{~h}$, control or plasmid group. ${ }^{\#} \mathrm{P}<0.05$, ${ }^{\# \#} \mathrm{P}<0.01$ vs. the ROMO1 KO group. ROMO1, reactive oxygen species modulator 1; TGF, transforming growth factor; KO, knockout; p, phosphorylation; Smad, mothers against decapentaplegic homolog; E, epithelial; NAC, NAC, N-acetyl-L-cysteine. 
with hemorrhage in the pulmonary vein. Similar to in the kidney, improvement was observed following treatment.

Oxidative stress-induced increase in ROMO1 expression and TGF- $\beta 1$ pathway activation is suppressed following ROMOI $K O$. HK-2 cells were treated with $\mathrm{H}_{2} \mathrm{O}_{2}$ to cause oxidative stress and the expression of ROMO1 was measured $0.5,1$ and $2 \mathrm{~h}$ treatment with $\mathrm{H}_{2} \mathrm{O}_{2}$. As demonstrated in Fig. 5A, the expression of ROMO1 was upregulated following $\mathrm{H}_{2} \mathrm{O}_{2}$ treatment in a time-dependent manner. Furthermore, a ROMO1-KO cell model was established, as presented in Fig. 5B, which demonstrated that the level of ROMO1 was decreased in the ROMO1-KO cell model when compared with the control.

As presented in Fig. 5C, the level of ROMO1 was markedly increased by oxidative stress following transfection with the control plasmid; however, ROMO1 expression was decreased following ROMO1 KO. Expression of ROMO1 was downregulated by treatment with NAC and the effect of NAC was improved when ROMO1 was knocked down. Furthermore, the alteration in TGF- $\beta 1$ signaling and EMT was investigated. The present study demonstrated that the expression of TGF- $\beta 1$, Smad2/3 and $p-S m a d 2 / 3$ was increased by $\mathrm{H}_{2} \mathrm{O}_{2}$, while their expression was suppressed when ROMO1 was knocked down. With regard to EMT, the expression of vimentin was increased by $\mathrm{H}_{2} \mathrm{O}_{2}$, while it was downregulated by ROMO1 KO. However, the expression of E-cadherin exhibited the opposite phenomenon asvimentin, as presented in Fig. 5C.

\section{Discussion}

To the best of the authors' knowledge, the present study was the first to use a UUO rat model to investigate the association between ROMO1 and RIF, demonstrating that increased ROMO1 expression causes an upregulation in ROS, and leads to TGF- $\beta 1$ signaling pathway activity, ECM generation and increased EMT.

RIF serves an important role in the development of renal disease, which is associated with the prognosis of chronic kidney disease, and is an essential step in the development of end-stage renal failure (18). In the present study, a UUO model was established to evaluate the influence of ROMO1 in the regulation of ROS, TGF- $\beta$ pathway activation and RIF occurrence. Serious renal dysfunction (RD) was observed in the present study. Creatinine clearance is widely utilized to measure renal function $(19,20) \mathrm{BUN} /$ creatinine clearance helps to differentiate chronic kidney disease from heart failure-induced RD (21). UA is upregulated under the condition of renal insufficiency (22). In the present study, it was demonstrated that creatinine clearance was decreased, while BUN and UA were increased in the UUO model. However, the symptoms were improved to different degrees following treatment with oxidation inhibitors. Apo (23) and NAC (24) are regarded as an antioxidants in vascular cells. Tempol assists in the clearance of ROS levels (25). Furthermore, it was also observed that the morphological damage in the kidney and lung may be relieved following treatment with oxidation inhibitors to a certain extent. Consequently, it may be inferred that a downregulation in kidney-associated ROS may reduce the occurrence of kidney damage by different mechanisms.

ROMO1 is an ROS regulatory factor, which is encoded on the mitochondrion and helps to increase the expression of intracellular ROS $(26,27)$. Increased ROS results in oxidative damage in a variety of signaling pathways, which causes serious diseases, including diabetes, neural degenerative diseases and tumors $(28,29)$. A previous study indicated that a physiological concentration of ROS is harmless. However, dysfunction the kidney may lead to increased ROS and imbalanced hemodynamics (30). In the present study, increased excretion of ROMO1 and ROS was not observed in blood serum in the UUO model. Furthermore, $\mathrm{H}_{2} \mathrm{O}_{2}$ is regarded as an important element that influences cellular proliferation (31). In the present study, the expression of ROMO1 was upregulated when the HK-2 cell line was treated with $\mathrm{H}_{2} \mathrm{O}_{2}$. Therefore, it may be concluded that oxidative stress increases ROMO1 expression. Overexpression was alleviated following treatment with oxidation inhibitors. Consequently, there is a relationship between ROMO1 and ROS in RIF.

RIF is a complicated mechanism which is accompanied by multiple cytokines, the most significant factor being TGF- $\beta 1$ in EMT $(30,32,33)$. The expression of TGF- $\beta 1$ is weak in normal renal tissues, while it is upregulated in RIF and stimulates ECM production, the primary factor that leads to RIF is ROS generation $(30,32,33)$. Furthermore, there is a mutual positive association between activated TGF- $\beta 1$ and $\mathrm{FN}$, in that $\mathrm{FN}$ is increased when TGF- $\beta 1$ is upregulated (34). FN is an important element in ECM that helps to stick auxiliary cells and other ECM molecules together $(30,32,33)$. The stimulated TGF- $\beta$ signaling pathway regulates TGF- $\beta / \mathrm{Smad}$ through the phosphorylation of Smad2 and Smad3, which causes a decrease in epithelial biomarkers (E-cadherin) and a rise in interstitial biomarkers, including vimentin, $\mathrm{N}$-cadherin. These situations result in a clear fiber phenotype and a lack of cell polarity $(35,36)$. In the present study, evidence was provided that ROMO1 expression increases during UUO, which stimulates ECM, FN production and increased expression of TGF- $\beta 1$ through the phosphorylation of Smad2/3, which causes RIF. Similar effects in the expression of ROMO1, TGF- $\beta$ and EMT were observed in the HK-2 cell line treated with $\mathrm{H}_{2} \mathrm{O}_{2}$. These effects were relieved when ROMO1 was knocked down or following treatment with oxidation inhibitors. Therefore, it was speculated that the effect of oxidative stress was suppressed to a certain extent when ROMO1 was knocked down, through the TGF- $\beta$ signaling pathway.

In conclusion, the TGF- $\beta$ signaling pathway was demonstrated to be activated by the production of ROMO1, which then induces RIF exaggeration. The associated factors of TGF- $\beta$, including Smad2/3 and ECM proteins, were triggered or generated during UUO and in the HK-2 cells. However, the symptoms were relieved following oxidation inhibitor intervention and ROMO1 KO. Consequently, it may be concluded that the regulation of ROMO1 provides further insight into RIF and its treatment.

\section{References}

1. Yang J and Liu Y: Dissection of key events in tubular epithelial to myofibroblast transition and its implications in renal interstitial fibrosis. Am J Pathol 159: 1465-1475, 2001.

2. Manotham K, Tanaka T, Matsumoto M, Ohse T, Inagi R, Miyata T, Kurokawa K, Fujita T, Ingelfinger JR and Nangaku M: Transdifferentiation of cultured tubular cells induced by hypoxia. Kidney Int 65: 871-880, 2004. 
3. Galichon P and Hertig A: Epithelial to mesenchymal transition as a biomarker in renal fibrosis: Are we ready for the bedside? Fibrogenesis Tissue Repair 4: 11, 2011.

4. Liu Y: Epithelial to mesenchymal transition in renal fibrogenesis: Pathologic significance, molecular mechanism, and therapeutic intervention. J Am Soc Nephrol 15: 1-12, 2004.

5. Lee SH, Min JW, Lee JS, Kim CH, Yoo YD, Lee EJ, Min KH, Hur GY, Lee SH, Lee SY, et al: Reactive oxygen species modulator 1 (Romo1) overexpression is an independent predictor of poor survival in NSCLC patients who undergo surgical resection. Lung Cancer 87: 45-52, 2015.

6. Shyamsunder P, Verma RS and Lyakhovich A: ROMO1 regulates RedOx states and serves as an inducer of NF- $\kappa \mathrm{B}$-driven EMT factors in Fanconi anemia. Cancer Lett 361: 33-38, 2015.

7. Hsieh HL, Wang HH, Wu WB, Chu PJ and Yang CM: Transforming growth factor- $\beta 1$ induces matrix metalloproteinase- 9 and cel migration in astrocytes: Roles of ROS-dependent ERK- and JNK-NF-кB pathways. J Neuroinflammation 7: 88, 2010.

8. Kawada N, Moriyama T, Ando A, Fukunaga M, Miyata T, Kurokawa K, Imai E and Hori M: Increased oxidative stress in mouse kidneys with unilateral ureteral obstruction. Kidney Int 56: 1004-1013, 1999.

9. Diekmann D, Abo A, Johnston C, Segal AW and Hall A: Interaction of Rac with p67phox and regulation of phagocytic NADPH oxidase activity. Science 265: 531-533, 1994.

10. Yagisawa M, Yuo A, Yonemaru M,Imajoh-Ohmi S, Kanegasaki S, Yazaki Y and Takaku F: Superoxide release and NADPH oxidase components in mature human phagocytes: Correlation between functional capacity and amount of functional proteins. Biochem Biophys Res Commun 228: 510-516, 1996.

11. Burdon RH, Alliangana D and Gill V: Hydrogen peroxide and the proliferation of Bhk-21 cells. Free Radic Res 23: 471-486, 1995.

12. Giraud S, Favreau F, Chatauret N, Thuillier R, Maiga S and Hauet T: Contribution of large pig for renal ischemia-reperfusion and transplantation studies: The preclinical model. J Biomed Biotechnol 2011: 532127, 2011.

13. Giles AR: Guidelines for the use of animals in biomedical research. Thromb Haemost 58: 1078-1084, 1987.

14. Cho A and Seok SH: Ethical guidelines for use of experimental animals in biomedical research. J Bacteriol Virol 43: 18-26, 2013

15. Livak KJ and Schmittgen TD: Analysis of relative gene expression data using real-time quantitative PCR and the 2(-Delta Delta C(T)) Method. Methods 25: 402-408, 2001.

16. Yoon YS, Lee JH, Hwang SC, Choi KS and Yoon G: TGF beta1 induces prolonged mitochondrial ROS generation through decreased complex IV activity with senescent arrest in Mv1Lu cells. Oncogene 24: 1895-1903, 2005.

17. Fischer AH, Jacobson KA, Rose J and Zeller R: Hematoxylin and eosin staining of tissue and cell sections. CSH Protoc 2008: pdb. prot4986, 2008.

18. Eddy AA: Molecular basis of renal fibrosis. Pediatr Nephrol 15: 290-301, 2000

19. Narva AS and Bilous RW: Laboratory assessment of diabetic kidney disease. Diabetes Spectr 28: 162-166, 2015.

20. Perrone RD, Madias NE and Levey AS: Serum creatinine as an index of renal function: New insights into old concepts. Clin Chem 38: 1933-1953, 1992.
21. Brisco MA, Coca SG, Chen J, Owens AT, McCauley BD, Kimmel SE and Testani JM: Blood urea nitrogen/creatinine ratio identifies a high-risk but potentially reversible form of renal dysfunction in patients with decompensated heart failure. Circ Heart Fail 6: 233-239, 2013

22. Leyva F, Anker S, Swan JW, Godsland IF, Wingrove CS, Chua TP, Stevenson JC and Coats AJ: Serum uric acid as an index of impaired oxidative metabolism in chronic heart failure. Eur Heart J 18: 858-865, 1997.

23. Heumüller S, Wind S, Barbosa-Sicard E, Schmidt HH, Busse R, Schröder K and Brandes RP: Apocynin is not an inhibitor of vascular NADPH oxidases but an antioxidant. Hypertension 51: 211-217, 2008.

24. Ferrari G, Yan CY and Greene LA: N-acetylcysteine (D- and L-stereoisomers) prevents apoptotic death of neuronal cells. J Neurosci 15: 2857-2866, 1995.

25. Dikalov S, Grigor'ev IA, Voinov M and Bassenge E: Detection of superoxide radicals and peroxynitrite by 1-hydroxy-4-phosphonooxy-2,2,6,6-tetramethylpiperidine: Quantification of extracellular superoxide radicals formation. Biochem Biophys Res Commun 248: 211-215, 1998.

26. Chung YM, Lee SB, Kim HJ, Park SH, Kim JJ, Chung JS and Yoo YD: Replicative senescence induced by Romol-derived reactive oxygen species. J Biol Chem 283: 33763-33771, 2008.

27. Lee SB, Kim JJ, Kim TW, Kim BS, Lee MS and Yoo YD: Serum deprivation-induced reactive oxygen species production is mediated by Romo1. Apoptosis 15: 204-218, 2010.

28. Apel K and Hirt H: Reactive oxygen species: Metabolism, oxidative stress, and signal transduction. Annu Rev Plant Biol 55: 373-399, 2004

29. Murphy MP: How mitochondria produce reactive oxygen species. Biochem J 417: 1-13, 2009.

30. Nath KA and Norby SM: Reactive oxygen species and acute renal failure. Am J Med 109: 665-678, 2000.

31. Ma L, Zhu WZ, Liu TT, Fu HL, Liu ZJ, Yang BW, Song TY and Li GR: H2O2 inhibits proliferation and mediates suppression of migration via DLC1/RhoA signaling in cancer cells. Asian Pac J Cancer Prev 16: 1637-1642, 2015.

32. Park SA, Kim MJ, Park SY, Kim JS, Lee SJ, Woo HA, Kim DK, Nam JS and Sheen YY: EW-7197 inhibits hepatic, renal, and pulmonary fibrosis by blocking TGF- $\beta /$ Smad and ROS signaling. Cell Mol Life Sci 72: 2023-2039, 2015.

33. Yang KL, Chang WT, Chuang CC, Hung KC and Li EI: Antagonizing TGF-beta induced liver fibrosis by a retinoic acid derivative through regulation of ROS and calcium influx. Biochem Biophys Res Commun 365: 484-489, 2008.

34. Peng F, Zhang B, Wu D, Ingram AJ, Gao B and Krepinsky JC: TGFbeta-induced RhoA activation and fibronectin production in mesangial cells require caveolae. Am J Physiol Renal Physiol 295: F153-F164, 2008.

35. Mizutani A, Koinuma D, Tsutsumi S, Kamimura N, Morikawa M, Suzuki HI, Imamura T, Miyazono K and Aburatani H: Cell type-specific target selection by combinatorial binding of Smad2/3 proteins and hepatocyte nuclear factor 4alpha in HepG2 cells. J Biol Chem 286: 29848-29860, 2011.

36. Yoshida $\mathrm{K}$ and Matsuzaki K: Differential regulation of TGF- $\beta /$ Smad signaling in hepatic stellate cells between acute and chronic liver injuries. Front Physiol 3: 53, 2012. 